\section{LA GRANJA:}

REVISTA DE

CIENCIAS DE LA VIDA

pISSN:1390-3799; eISSN:1390-8596

http:/ / doi.org/10.17163/lgr.n34.2021.07
Artículo científico / Scientific paper

SILVICULTURA

(c) (1) (5)(2)

\title{
PROPAGACIÓN SEXUAL Y ASEXUAL DE BROSIMUM ALICASTRUM SWARTZ EN CAMPECHE, MÉXICO
}

\author{
SEXUAL AND ASEXUAL PROPAGATION OF BROSIMUM ALICASTRUM \\ SWARTZ IN CAMPECHE, MEXICO
}
Alberto Santillán-Fernández ${ }^{* 1}{ }_{\circledast \infty}$, Orlando Valentín Santiago-Santes ${ }^{2}{ }^{\oplus}$, Ezequiel Espinosa-Grande ${ }^{1}{ }^{1}$, Zulema Guadalupe Huicab-Pech $^{3} \oplus$, Francisco Alfonso

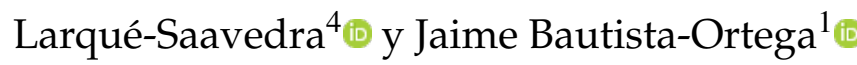

\footnotetext{
${ }^{1}$ Colegio de Postgraduados Campus Campeche. Código Postal 24400, Champotón, Campeche, México.

${ }^{2}$ Ingeniería Forestal. Instituto Tecnológico Superior de Venustiano Carranza. Código Postal 73049, Venustiano Carranza, Puebla, México.

${ }^{3}$ Investigador independiente. Código Postal 24020, Campeche, Campeche, México.

${ }^{4}$ Unidad de Recursos Naturales. Centro de Investigación Científica de Yucatán. Código Postal 97205, Mérida, Yucatán, México.

*Autor para correspondencia: asantillanf@conacyt.mx
}

Manuscrito recibido el 3 de julio de 2020. Aceptado, tras revisión, el 1 de marzo de 2021. Publicado el 1 de septiembre de 2021.

\begin{abstract}
Resumen
Brosimum alicastrum es una especie arbórea en México con amplio potencial para la alimentación animal y humana, que se distribuye de manera natural con nulo manejo silvícola, por lo que existe poca información sobre los métodos de propagación de la especie. El objetivo de este trabajo fue analizar la producción científica reportada sobre B. alicastrum mediante minería de textos para conocer las técnicas que existen sobre su propagación; y evaluar en vivero la calidad de plántulas obtenidas por métodos de propagación sexual y asexual (estacas, acodos e injertos) mediante diseños experimentales. Se encontraron 550 artículos científicos sobre B. alicastrum, las disciplinas donde se publicaron fueron: Ecología (44,18\%), Botánica (13,27\%), Ciencias Forestales (11,27\%, de los cuales el 2,54\% trabajó propagación en vivero), Zoología (11,09\%), Agricultura (9,64\%), Antropología (5,45\%) y otras $(5,10 \%)$. Respecto al método de propagación por semilla, la mejor calidad de plántula se asoció con sustratos de baja porosidad (tierra de monte) y con contenedores con diámetros grandes $(36 \mathrm{~cm})$. Para el caso de la propagación asexual por acodos, cuando se empleó turba como sustrato se obtuvo $90 \%$ de sobrevivencia, y por injerto de enchape lateral se encontró $75 \%$ de prendimiento. En virtud de la poca investigación que existe sobre la propagación de la especie se recomienda que la selección de la técnica de propagación esté en función de la finalidad de la plántula. Las técnicas asexuales de injerto y acodo pueden ser más eficientes en caso de requerir acortar los ciclos de producción de la semilla de B. alicastrum.
\end{abstract}

Palabras clave: Ramón, silvicultura, vivero forestal, injerto, enraizamiento de estacas, acodo aéreo 


\begin{abstract}
Brosimum alicastrum is a tree species in Mexico with wide potential for animal and human food, which is distributed naturally with no silvicultural management, so there is little information on the propagation methods of the species. The objective of this work was to analyze the scientific research published on B. alicastrum, through literature review to know the techniques that exist on its propagation. In addition, the quality of the seedling obtained by sexual propagation and asexual methods (cuttings, layers and grafts) was evaluated in the nursery, by means of experimental designs. 550 scientific articles on B. alicastrum were found, the disciplines where they were published were: Ecology $(44.18 \%)$, Botany $(13.27 \%)$, Forest Sciences $(11.27 \%$, of which $2.54 \%$ worked propagation in the nursery), Zoology $(11.09 \%)$, Agriculture $(9.64 \%)$, Anthropology $(5.45 \%)$ and others $(5.10 \%)$. Regarding the seed propagation method, the best seedling quality was associated with low porosity substrates (bush soil) and containers with large diameters $(36 \mathrm{~cm})$. In the case of asexual propagation, with the layering method when peat was used as the substrate $90 \%$ survival was obtained, and by lateral grafting technique $75 \%$ yield was found. Due to the little research that exists on the propagation of the species, it is recommended that the selection of the propagation technique is based on the purpose of the seedling; if it is required to shorten the seed production cycles of B. alicastrum the asexual techniques grafting and layering can be more efficient.
\end{abstract}

Keywords: Ramon, forestry, forest nursery, graft, rooting of cuttings, air layering.

Forma sugerida de citar: Santillán, A., Santiago, O., Espinosa, E., Huicab, Z., Larqué, F. y Bautista, J. (2021). Propagación sexual y asexual de Brosimum alicastrum Swartz en Campeche, México. La Granja: Revista de Ciencias de la Vida. Vol. 34(2):105-116. http:/ / doi.org/10.17163/lgr. n34.2021.07.

IDs Orcid:

Alberto Santillán-Fernández: https:/ / orcid.org/0000-0001-9465-1979

Orlando Valentín Santiago-Santes: https:/ / orcid.org/0000-0003-2703-3737

Ezequiel Espinosa-Grande: https:/ /orcid.org/0000-0003-2181-1876

Zulema Guadalupe Huicab-Pech: https:/ / orcid.org/0000-0003-1648-5172

Francisco Alfonso Larqué-Saavedra: https:/ / orcid.org/0000-0001-9462-5682

Jaime Bautista-Ortega: https:/ / orcid.org/0000-0002-3763-8986 


\section{Introducción}

Brosimum alicastrum Swartz, conocido comúnmente como Ramón, es un árbol originario de Mesoamérica y el Caribe con amplia distribución en México (Peters y Pardo, 1982). Es apreciado por poseer follaje con alto contenido nutritivo, principalmente para el ganado vacuno y caprino y también por su disponibilidad durante la época de sequía (Hernández, Vergara y Larqué, 2014; Rojas y col., 2017). Ramón representa un elemento ecológicamente importante en la composición florística de las selvas baja y mediana del sur de México (Gutiérrez y Dirzo, 2009).

Ramón tiene la capacidad de producir cuatro veces más alimento y 10 veces más proteína por hectárea que el maíz, sin causar daños al ambiente; además, su semilla, follaje, látex y madera tienen un alto potencial económico, tanto para la alimentación (animal y humana) como para usos medicinales y culturales (Ramírez y col., 2017; Domínguez y col., 2019). Sin embargo, aun con toda esta importancia, en la actualidad la especie se distribuye mayormente de manera natural, con prácticamente nulo manejo silvícola (Vergara y col., 2014).

En el establecimiento de plantaciones se requieren árboles con características fenotípicas y genotípicas sobresalientes, por lo que el método de propagación para la obtención del germoplasma es clave para un buen manejo silvícola (Hernández, Vergara y Larqué, 2015). La propagación puede ser de manera sexual o asexual (Molina y col., 2015).

En la reproducción sexual de los árboles, los progenitores pueden heredar características deseables como indeseables (Molina y col., 2015); mientras que en la propagación vegetativa se puede obtener germoplasma con información genética y rasgos de importancia económica en períodos más cortos, con la desventaja de que se reduce la diversidad genética de la especie por la uniformidad de la descendencia (Bailey, Bímová y Mandák, 2009). En ambos casos el manejo en vivero se refleja en la calidad de la plántula producida, a través de sus características morfológicas y de su capacidad de adaptación al sitio de plantación (Rueda y col., 2014).

Las técnicas tradicionales de propagación asexual en especies forestales son estacas, acodos e injertos (Pardo, Verdugo y Hernández, 2002). Con el avance de la biotecnología la propagación in vitro se ha vuelto una alternativa para aquellas especies con alto valor comercial y de difícil propagación por técnicas tradicionales (Bailey, Bímová y Mandák, 2009). Sin embargo, la principal limitante de la propagación vegetativa es el bajo porcentaje de multiplicación, por ello es importante ampliar el número de especies a propagar y mejorar la técnica, ya que las plantas reproducidas vegetativamente presentan mejor manejo silvícola (Sampayo y col., 2016).

La investigación sobre técnicas de propagación en la especie $B$. alicastrum es casi nula, y la que existe está reportada en su mayoría en formato de manual, sin ningún rigor científico (Gillespie, Bocanegra y Jimenez, 2004; Molina y col., 2015). Dada la importancia de la especie en la península de Yucatán como un recurso vegetal alternativo para la alimentación animal y humana en el contexto de seguridad alimentaria y cambio climático (Ramírez y col., 2017), toma valor la investigación que se desarrolle en torno al manejo silvícola de la especie (Hernández, Vergara y Larqué, 2015).

Bajo este contexto el objetivo de este trabajo fue analizar la producción científica reportada sobre Brosimum alicastrum Swartz, mediante minería de textos para conocer las técnicas que existen sobre su propagación. Además de evaluar en vivero, mediante diseños experimentales, la calidad de plántulas obtenidas por métodos de propagación sexual y asexual (estacas, acodos e injertos).

\section{Materiales y Métodos}

\section{1 Área de estudio}

El trabajo se realizó en el vivero experimental ubicado en las instalaciones del Colegio de Postgraduados campus Campeche (Champotón, Campeche). El material vegetativo de $B$. alicastrum fue recolectado en diferentes localidades aledañas al centro de investigación (Figura 1). La región se caracteriza por una cobertura de selva media subperenifolia y selva alta perenifolia, con suelos arcillosos y precipitaciones de 600 a $4000 \mathrm{~mm}$, con épocas de estiaje de tres a siete meses, temperatura media anual de 18 ${ }^{\circ} \mathrm{C}$ a $27^{\circ} \mathrm{C}$ y altitudes de 20 a $1000 \mathrm{msnm}$. Las principales actividades económicas de esta región son 
la ganadería y el cultivo de maíz (Zea mays L.), frijol (Phaseolus vulgaris L.), chihua (Cucurbita argyrosperma H.) y caña de azúcar (Saccharum officinarum L.) (White y Hood, 2004). Destaca que en el periodo de estiaje el principal alimento para el ganado es el árbol Ramón (Góngora y col., 2016).

\subsection{Minería de textos y análisis bibliomé- trico}

Para conocer las técnicas que existen sobre la propagación de $B$. alicastrum se consideraron los artículos científicos sobre la especie disponibles en las principales casas editoriales (Elsevier, Springer y Scopus) y sitios web (Latindex, Scielo, Redalyc, Thomson-Reuters, Periodica, Doaj, Google Scholar y Conricyt). La palabra clave utilizada en la búsqueda fue Brosimum alicastrum, identificándola en los títulos y palabras clave de las publicaciones.
Las variables que se analizaron de cada artículo fueron: año para conocer la temporalidad de las investigaciones, disciplina de estudio para determinar el área de conocimiento donde más investigación se ha desarrollado, y autores para conocer la red de actores involucrados en la investigación. La información se sistematizó en una hoja de cálculo. Con ayuda del complemento RcmdrPlugin.temis del software estadístico R (Bouchet y Bastin, 2013) se obtuvo la temporalidad de las investigaciones y la frecuencia de las publicaciones por disciplina de estudio.

Con el software Sci2tool (Börner, 2011) se estableció la red de autores. A los autores con más publicaciones en la red se les asoció el área de conocimiento donde han desarrollado sus investigaciones. La sintaxis empleada en el software Sci2tool fue $E x$ tract bipartite Network, para su visualización se recurrió al software Gephi (Jacomy y col., 2014).

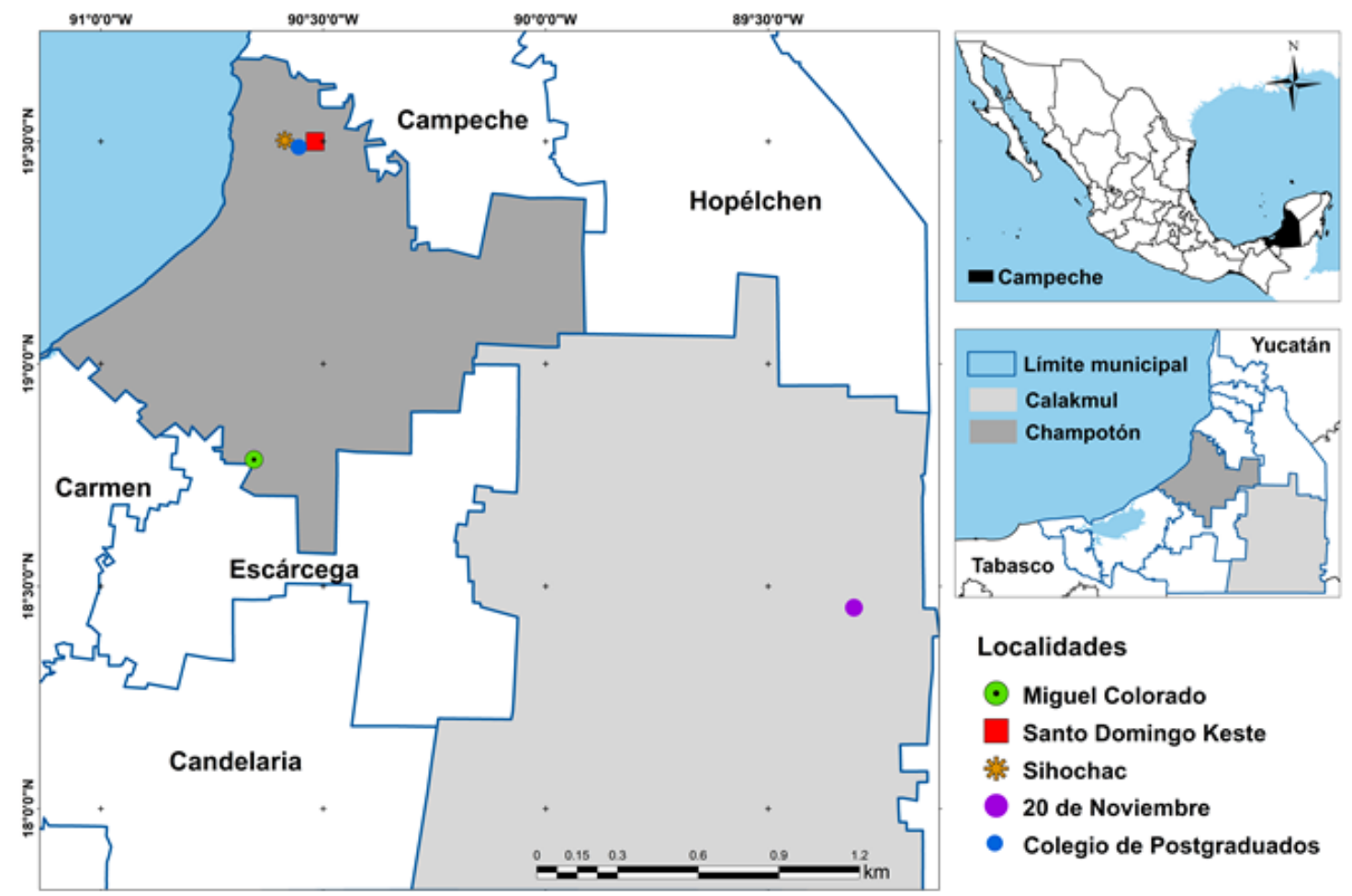

Figura 1. Ubicación espacial de las localidades donde se recolectó el material vegetativo y donde se realizaron los experimentos para evaluar la propagación de Brosimun alicastrum Swartz en Campeche, México. 


\subsection{Propagación sexual}

En marzo de 2019 se recolectó semilla de Ramón en la localidad 20 de noviembre (Calakmul, Campeche). Para la recolecta de semilla se siguió la metodología descrita por Vallejos y col. (2010), y se seleccionaron los árboles con las mejores características fenotípicas (dasométricas) con una distancia mínima entre árboles seleccionados de $100 \mathrm{~m}$. El germoplasma se almacenó en bolsas esterilizadas de plástico que fueron trasladadas a las instalaciones del Colegio de Postgraduados, campus Campeche (Champotón, Campeche), México. Las semillas fueron sometidas a un análisis de pureza y una prueba de viabilidad con cloruro de tetrazolio $\AA$ al $0,5 \%$, conforme la metodología descrita por Orantes y col. (2013). Se evaluó en un vivero forestal rustico de abril a junio de 2020, donde se estableció un diseño experimetal completamente al azar con arreglo factorial. Los factores analizados fueron: 1) tipo de contenedor: tubetes $(36$ y $21 \mathrm{~cm}$ de diámetro) y bolsa de plástico (36 y $21 \mathrm{~cm}$ de diámetro), y 2) sustrato: (peat moss $50 \%+$ agrolita $25 \%+$ perlita $25 \%$ ) y (tierra de monte $60 \%$ + lumbricompost $40 \%)$.

La mezcla (peat moss $50 \%$ + agrolita $25 \%$ + perlita $25 \%$ ) se caracterizó por un $\mathrm{pH}$ ligeramente acido con alta porosidad y capacidad de retención de humedad; mientras que la mezcla (tierra de monte $60 \%$ + lumbricompost $40 \%$ ) presentó un $\mathrm{pH}$ neutro con baja porosidad y alta capacidad de retención de agua (Pérez de la Cruz y col., 2012). Para los ocho tratamientos, se aplicó un riego manual cada tres días a las unidades experimentales, conforme la retención de humedad de los sustratos utilizados (Del Amo y col., 2002).

A los 30 días de establecido el diseño experimental, se evaluó el porcentaje de germinación por tratamiento. Con un análisis de varianza y pruebas de medias por Tukey $(\alpha=0,05)$ se establecieron las diferencias estadísticas por tratamiento a los tres meses de establecido el experimento para las variables: altura $(\mathrm{cm})$, numero de hojas, diámetro de tallo $(\mathrm{cm})$, biomasa seca del tallo $(\mathrm{kg})$, ancho de la raíz $(\mathrm{cm})$, largo de la raíz $(\mathrm{cm})$ y biomasa seca de la raíz $(\mathrm{kg})$. La biomasa seca de la raíz y tallo se obtuvo en una balanza analítica después de extraer el material vegetativo de una estufa con circulación de aire forzado a $70{ }^{\circ} \mathrm{C}$ durante 24 horas.

\subsection{Propagación asexual}

Se probaron tres técnicas de propagación asexual: estacas, acodos e injertos. Para el caso de las estacas, el material vegetal se recolectó en marzo de 2019 en la comunidad Miguel Colorado (Champotón, Campeche). Mediante un recorrido en campo se seleccionaron árboles sanos, vigorosos, libres de plagas, con frutos en el momento de la recolecta, y rectos sin bifurcación. El material vegetal fue envuelto en papel periódico húmedo para evitar que se deshidratara durante su traslado al vivero del Colegio de Postgraduados, campus Campeche.

Las estacas fueron colocadas en bolsas de polietileno con sustrato tradicional de vivero (peat moss $50 \%$, agrolita $25 \%$ y perlita $25 \%$ ). Se evaluaron brotes y raíces en las estacas a los tres meses (abril-junio) mediante un diseño experimental completamente al azar con arreglo factorial. Los factores considerados fueron: tipo de enraizador (Auxina, Fortimax, Raidzone Plus y Magic Plus), y posición de estaca (basal, intermedia y apical). Para los 12 tratamientos se aplicó un riego manual cada tres días a las unidades experimentales. Las variables analizadas fueron número y longitud $(\mathrm{cm})$ de los brotes aéreos, y biomasa seca y fresca de raíces y tallos $(\mathrm{kg})$, altura vertical $(\mathrm{cm})$ y horizontal $(\mathrm{cm})$ de la raíz.

En mayo de 2019 se desarrolló el experimento de propagación in vivo por acodos a árboles jóvenes de Ramón (menos de 5 años) en la localidad de Sihochac (Champotón, Campeche). Mediante un recorrido en campo, se seleccionaron arboles sanos, vigorosos, libres de plagas y rectos sin bifurcación. Se consideró un diseño experimental completamente al azar con tres tratamientos $(\mathrm{T})$ y 30 repeticiones por tratamiento: T1 (Auxina + Turba), T2 (Auxina + Vermiculita) y T3 (Auxina + Perlita). Las variables analizadas fueron: porcentaje de sobrevivencia a los tres meses, y número, longitud $(\mathrm{cm})$ y biomasa seca $(\mathrm{kg})$ de las raíces.

En julio de 2019 en la localidad de Santo Domingo Keste (Champotón, Campeche) se llevó a cabo el experimento de propagación in vivo por injerto a arboles jóvenes de Ramón menores a un año, con material vegetativo de árboles de la misma especie no menores a cinco años y con capacidad de producir frutos. Para la selección de los árboles patrón y de donde se obtuvieron las varetas a injertar, 
se seleccionaron árboles sanos, vigorosos, libres de plagas y rectos sin bifurcación.

Las técnicas de injerto utilizadas fueron: hendidura, yema, enchape lateral e inglés simple. Se estableció un diseño experimental completamente al azar, donde se tomó a cada técnica de injerto como un tratamiento con 20 repeticiones por tratamiento. Las variables analizadas fueron porcentaje de prendimiento a los tres meses. Además de altura $(\mathrm{cm})$, diámetro $(\mathrm{cm})$, número de hojas y número de brotes del material vegetativo injertado. Para establecer las diferencias estadísticas por tratamientos y variables analizadas, se efectuó un análisis de varianza y pruebas de medias por Tukey $(\alpha=0,05)$ tanto para el método de propagación por estacas como para acodos e injertos.

\section{Resultados y Discusión}

\subsection{Minería de textos y análisis bibliomé- trico}

De 1970 a 2019 se publicaron 550 artículos científicos donde la especie $B$. alicastrum apareció en el titulo o palabras clave. De acuerdo con De Granda y col. (2005) en el título se indica la temática precisa del trabajo y las palabras clave permiten ubicar en cualquier índice el tema tratado en el artículo. Las disciplinas que publicaron temas relacionados con la especie B. alicastrum fueron: Ecología $(44,18 \%)$, Botánica $(13,27 \%)$, Ciencias Forestales $(11,27 \%$, de los cuales el 2,54\% trabajó propagación en vivero), Zoología (11,09\%), Agricultura (9,64\%), Antropología $(5,45 \%)$ y otras $(5,10 \%)$.

En la Figura 2 se observa la red de autores de los 550 trabajos analizados. Se destacaron los autores con el mayor número de contribuciones por área de conocimiento. Ecología resultó ser la disciplina donde mayor investigación se ha desarrollado. Este aspecto ha sido reportado por Vergara y col. (2014) quienes coincidieron que para Ramón existe poca investigación sobre el manejo silvícola de la especie. Lo que constituye un área de oportunidad para el desarrollo de investigación, ya que de acuerdo con Ramírez y col. (2017) la especie tiene amplio potencial para la agroindustria de alimentos pecuarios, sobre todo en los sectores: porcino, bovino, ovino, avícola y acuícola en el marco de la seguridad alimentaria y cambio climático.

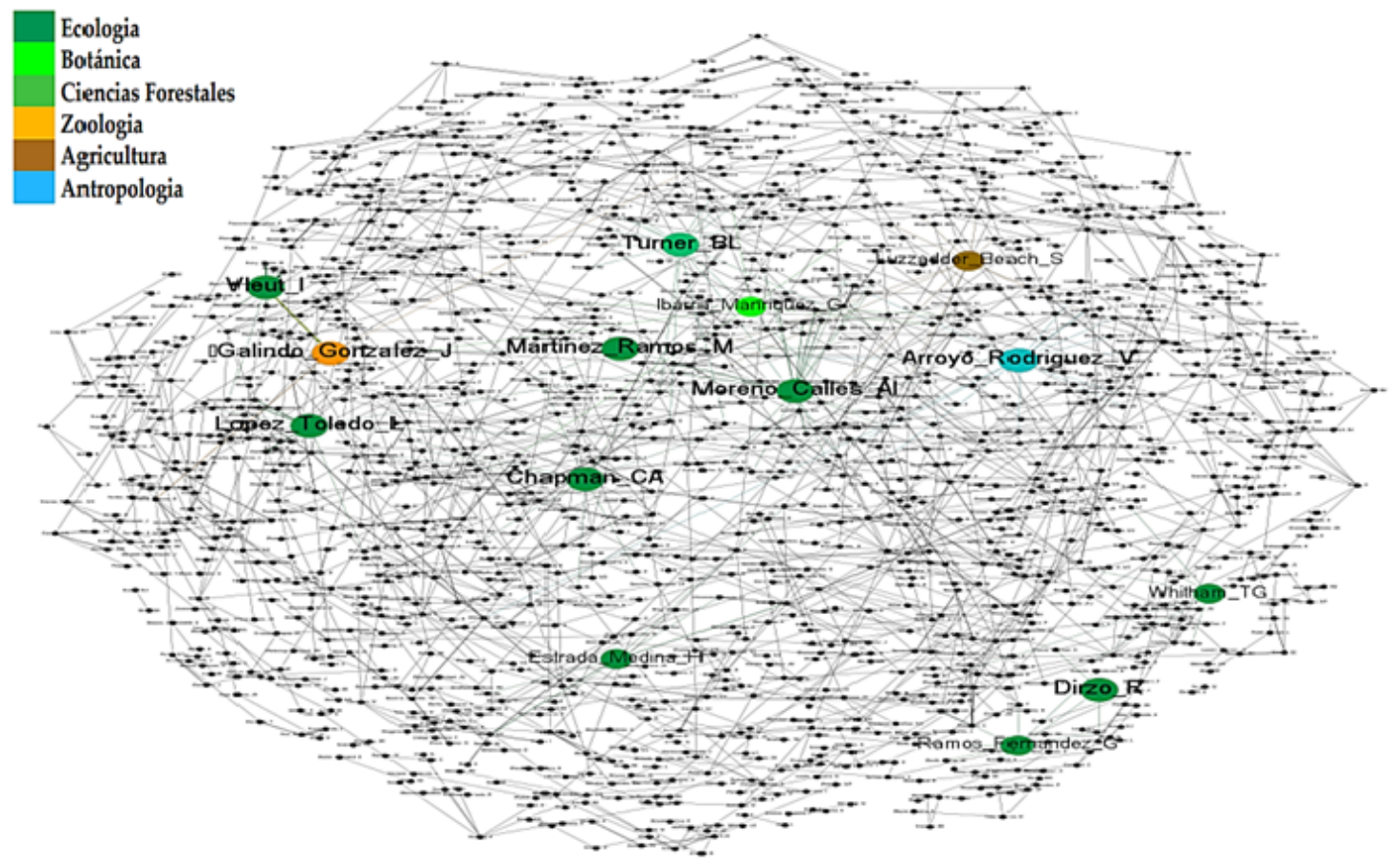

Figura 2. Red de autores de las disciplinas donde se ha desarrollado más investigación sobre Brosimum alicastrum Swartz. 
Hernández, Vergara y Larqué (2014) destacaron la importancia que las plantaciones con Ramón en el sureste mexicano tendrían para la agroindustria de alimentos pecuarios, al garantizar la materia prima de sus operaciones. De acuerdo con Vergara y col. (2014) el nulo manejo silvícola de la especie se comprende porque la industria en torno a $B$. alicastrum es emergente y la investigación actual se centra en evaluar sus propiedades y usos potenciales.

\subsection{Propagación Sexual}

Para la prueba de pureza, se analizaron 858 semillas de B. alicastrum con una biomasa total de 2128 g. El 91\% de las semillas $(785 ; 1944$ g) resultó no estar rota, vana, manchada y sin rastro de ataque de insectos (orificios y/o larvas). Sin embargo, el alto porcentaje de pureza contrastó con la viabilidad (potencial de germinación) de la semilla. Los resultados del contacto directo de las semillas con la solución de Tetrazolio® a temperatura ambiente y con luz limitada durante 24 horas arrojó en promedio para las cuatro repeticiones con 10 semillas por repetición, una viabilidad alta en un $20 \%$ de las semillas, media $(27,5 \%)$ y baja $(52,5 \%)$.

El bajo potencial de germinación de las semillas de B. alicastrum ha sido documentado por Gillespie, Bocanegra y Jimenez (2004) con valores inferiores al $50 \%$, lo que coincidió con los resultados obtenidos en esta investigación, pero contrastaron con lo reportado por Del Amo y col. (2002) con porcentajes de germinación superiores al $75 \%$. Estas diferencias se explicaron debido a que las semillas de Ramón son recalcitrantes (García, Roque y Méndez, 2012; Loría y Larqué, 20015), propiedad que las hace más sensibles a la deshidratación y rápida pérdida de viabilidad, lo que limita su almacenamiento con fines de propagación (Magnitskiy y Plaza, 2007).

La evaluación de la germinación a los 30 días post-siembra resultó en un 59,6\%, lo que coincidió con la baja viabilidad de la semilla obtenida en la prueba de Tetrazolio ${ }^{\circledR}$ (Tabla 1). Sin embargo, difirió con lo reportado por Laborde y Corrales (2012) quienes encontraron un 75\% de germinación cuando las semillas cayeron directamente del árbol al suelo. De acuerdo con Del Amo y col. (2002) el porcentaje de germinación de las semillas de B. alicastrum en vivero estuvo directamente relacionado con la temporalidad recolecta-siembra, por la propiedad recalcitrante de la semilla.

En la Tabla 1 se presentan los resultados del análisis de varianza y prueba de medias por Tukey $(\alpha=0,05)$ por tratamiento y variables evaluadas. Se observaron diferencias estadísticas entre los tratamientos utilizados $(\mathrm{P}<0,0001)$. La prueba de rangos múltiples de Tukey indicó que el tratamiento 6 (bolsa de $36 \mathrm{~cm}$ de diámetro con sustrato 2: tierra de monte $60 \%+$ lumbricompost $40 \%$ ) fue el que presentó los mayores valores para las características fenotípicas evaluadas.

El tipo de contenedor (tubete o bolsa de plástico) no mostró diferencias estadísticas. Este resultado coincidió con lo reportado por Luna, Landis y Dumroese (2012) quienes encontraron que el uso de bolsas de plástico como contenedor en vivero ocurre por términos económicos. El factor que si resultó estadísticamente significativo fue el tamaño del diámetro del contenedor. Los mayores valores para las características fenotípicas evaluadas se presentaron en aquellos contenedores de mayor diámetro $(36 \mathrm{~cm})$.

Este resultado contrastó con lo reportado por Pérez de la Cruz y col. (2012) quienes encontraron que en el primer mes en vivero las semillas de B. alicastrum presentaron un crecimiento más rapido en contenedores con diametros de $21 \mathrm{~cm}$. Sin embargo, de acuerdo con Luna, Landis y Dumroese (2012) los contenedores de mayor diámetro otorgaron mejores características en vivero a las especies de hojas grandes, por el espacio entre plantas, que les permitió aprovechar mejor la luz, calor, agua y nutrientes.

\subsection{Propagación Asexual}

La propagación por estacas no fue significativa, pues las unidades experimentales evaluadas en los diferentes tratamientos no lograron sobrevivir. Ninguna unidad experimental logró formación de raíces, y solo 7 de 240 presentaron brotes aéreos (Tabla 2). Sampayo y col. (2016) encontraron que fue común la presencia de brotes aéreos en especies forestales propagadas por estacas. Sin embargo, el éxito de esta técnica se midió por el porcentaje de enraizamiento logrado (Peralta y col., 2017). 


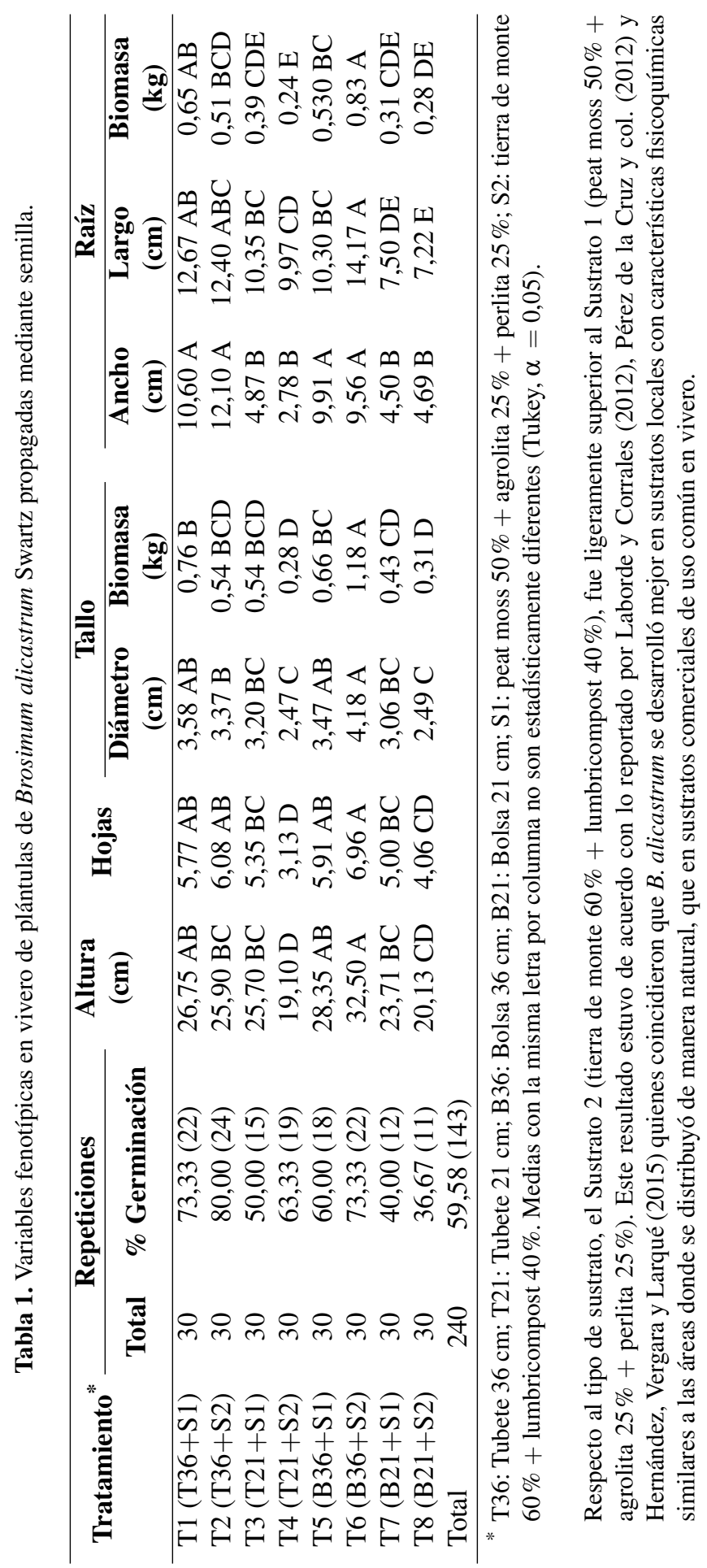


De acuerdo con González y col. (2019) en la propagación asexual por estacas de especies forestales, la obtención de resultados no significativos pudo ser producto de un inadecuado manejo en vivero, al tejido lignificado del material vegetal e incluso a la fecha de recolecta. No obstante, los resultados coincidieron con lo reportado por Vergara y col. (2014) y Molina y col. (2015) quienes postularon que la propagación por estacas para B. alicastrum fue una técnica no viable debido al tejido lignificado de la especie.

Los métodos de acodo e injerto si resultaron significativos y la prueba de medias por Tukey estableció diferencias estadísticas entre los tratamientos con un nivel de confianza del $95 \%(\alpha=0,05)$. Para el caso de acodos, el tratamiento con sustrato de turba presentó los mayores resultados (Tabla 3). En el caso de injerto, la técnica de enchape lateral fue la que resultó significativa con un prendimiento del $75 \%$ (15 de 20 repeticiones), superior al de hendidura $(20,00 \%$; cuatro de 20 repeticiones), yema $(0,00 \%$; cero de 20 repeticiones) e inglés simple $(10,00 \%$; dos de 20 repeticiones) (Tabla 4).

Molina y col. (2015) encontró en regiones de El Salvador que para la especie $B$. alicastrum el injerto constituyó la forma más viable de propagación asexual. Sin embargo, a diferencia de este estudio, no se consideraron tratamientos previos con hormonas de crecimiento a los materiales vegetativos injertados en esta investigación. En ambos estudios la técnica de enchape lateral fue la que mayor porcentaje de prendimiento logró: $75 \%$ en esta investigación y $42 \%$ para Molina y col. (2015).

Tabla 2. Variables fenotípicas del material vegetativo de Brosimun alicastrum propagado asexualmente mediante la técnica de estacas.

\begin{tabular}{lccccccccc}
\hline \multirow{2}{*}{ Tratamiento } & \multicolumn{2}{c}{ Repeticiones } & & \multicolumn{2}{c}{ Brotes aéreos } & & \multicolumn{2}{c}{ Raíz (Longitud: cm) } \\
\cline { 2 - 3 } & Total & Significativas & & Número & $\begin{array}{c}\text { Longitud } \\
(\mathbf{c m})\end{array}$ & & Biomasa & Vertical & Horizontal \\
\hline T1 (AU_BA) & 30 & 2 & & 3 & 0,5 & & 0 & 0 & 0 \\
T2 (AU_IN) & 30 & 2 & & 2 & 0,25 & & 0 & 0 & 0 \\
T3 (AU_AP) & 30 & 2 & & 3 & 3,93 & & 0 & 0 & 0 \\
T5 (FO_IN) & 30 & 1 & & 1 & 2,35 & & 0 & 0 & 0 \\
Otros** & 240 & 0 & & 0 & 0 & & 0 & 0 & 0 \\
\hline
\end{tabular}

Enraizador: Auxina (AU), Fortimax (FO), Raidzone Plus (RP), Magic Plus (MP). Posición de estaca: Basal (BA), Intermedia (IN), Apical (AP). ${ }^{* *}$ T4 (FO_BA), T6 (FO_AP), T7 (RP_BA), T8 (RP_IN), T9 (RP_AP), T10 (MP_BA), T11 (MP_IN), T12 (MP_AP).

Tabla 3. Variables fenotípicas del material vegetativo de Brosimun alicastrum Swartz propagado asexualmente mediante la técnica de acodos.

\begin{tabular}{lcccccc}
\hline \multirow{2}{*}{ Tratamiento } & \multicolumn{2}{c}{ Repeticiones } & & \multicolumn{3}{c}{ Raíz } \\
\cline { 2 - 3 } \cline { 5 - 7 } & Total & \% Sobrevivencia & & Número & $\begin{array}{c}\text { Longitud } \\
(\mathbf{c m})\end{array}$ & $\begin{array}{c}\text { Biomasa } \\
(\mathbf{k g})\end{array}$ \\
\hline T1 (Turba) & 30 & $90(27) \mathrm{A}$ & & $13,56 \mathrm{~A}$ & $11,99 \mathrm{~A}$ & $0,61 \mathrm{~A}$ \\
T2 (Vermiculita) & 30 & $80(24) \mathrm{B}$ & & $12,44 \mathrm{AB}$ & $8,55 \mathrm{~B}$ & $0,52 \mathrm{AB}$ \\
T3 (Perlita) & 30 & $80(24) \mathrm{B}$ & & $10,78 \mathrm{~B}$ & $9,05 \mathrm{~B}$ & $0,31 \mathrm{~B}$ \\
\hline
\end{tabular}

El enraizador utilizado para todos los tratamientos fue Auxina. Medias con la misma letra por columna no son estadísticamente diferentes (Tukey, $\alpha=0,05)$. El \% de Sobrevivencia se calculó por tratamiento, entre paréntesis se indica el número de repeticiones que sobrevivieron del total por tratamiento. 
Tabla 4. Variables fenotípicas del material vegetativo de Brosimun alicastrum Swartz propagado asexualmente mediante la técnica de injerto.

\begin{tabular}{cccccccc}
\hline \multirow{2}{*}{ Tipo de injerto } & \multicolumn{2}{c}{ Repeticiones } & & \multicolumn{4}{c}{ Material vegetativo injertado } \\
\cline { 2 - 3 } \cline { 5 - 7 } & Total & \% Prendimiento & & $\begin{array}{c}\text { Altura } \\
\text { (cm) }\end{array}$ & $\begin{array}{c}\text { Diámetro } \\
(\mathbf{c m})\end{array}$ & $\begin{array}{c}\text { Hojas } \\
(\text { número) }\end{array}$ & $\begin{array}{c}\text { Brotes } \\
\text { (número) }\end{array}$ \\
\hline Hendidura & 20 & $20,00(4) \mathrm{B}$ & & $8,75 \mathrm{AB}$ & $0,11 \mathrm{~B}$ & $8,72 \mathrm{~B}$ & $1,25 \mathrm{~B}$ \\
Yema & 20 & $0,00(0) \mathrm{C}$ & & $0,00 \mathrm{C}$ & $0,00 \mathrm{C}$ & $0,00 \mathrm{D}$ & $0,00 \mathrm{C}$ \\
Enchape lateral & 20 & $75,00(15) \mathrm{A}$ & & $10,35 \mathrm{~A}$ & $0,139 \mathrm{~A}$ & $15,59 \mathrm{~A}$ & $2,63 \mathrm{~A}$ \\
Inglés simple & 20 & $10,00(2) \mathrm{B}$ & & $5,54 \mathrm{~B}$ & $0,06 \mathrm{C}$ & $5,28 \mathrm{C}$ & $0,74 \mathrm{~B}$ \\
\hline
\end{tabular}

Medias con la misma letra por columna no son estadísticamente diferentes (Tukey, $\alpha=0,05$ ). El\% de Prendimiento se calculó por tipo de injerto, entre paréntesis se indica el número de repeticiones que presentaron prendimiento del total por tipo de injerto.

La diferencia en los porcentajes de prendimiento pudo explicarse por la condición juvenil de los materiales vegetativos empleados ( 5 años, con esquejes tiernos no lignificados) y patrón (1 año). De acuerdo con Alba y col. (2017) el porcentaje de prendimiento del material vegetativo injertado estuvo asociado al carácter juvenil de los materiales, por lo que cuanto más joven el individuo, más rápido y fácil su propagación. Lo que convierte al injerto en el método de propagación asexual más eficaz (Bailey, Bímová y Mandák, 2009).

Para el caso de la técnica de propagación por acodos, no se encontraron trabajos donde se reporte el empleo de esta técnica en la propagación asexual de B. alicastrum. De acuerdo con Vergara y col. (2014) el nulo manejo silvícola que presenta la especie, ha limitado el desarrollo de la investigación sobre sus formas de propagación.

\section{Conclusiones}

La producción científica encontrada sobre B. alicastrum se centró en analizar y describir aspectos de ecología y botánica de la especie; los trabajos sobre su propagación fueron escasos. Por lo que el desarrollo de investigación en torno a este tema es un área prioritaria para contribuir al manejo silvícola de la especie.

En el método de propagación por semilla, el porcentaje de germinación en vivero estuvo asociado con sustratos de $\mathrm{pH}$ neutro y baja porosidad, y la calidad de plántulas a contenedores con diámetros grandes $(36 \mathrm{~cm})$. La propagación asexual por estacas no fue factible; los mejores resultados se obtuvieron con el método acodos con un $90 \%$ de sobrevivencia, e injerto con la técnica enchape lateral con un $75 \%$ de prendimiento.

En virtud de la poca investigación que existe sobre la propagación de la especie, se recomienda que la selección de la técnica de propagación esté en función de la finalidad de la plántula, y si se requieren acortar los ciclos de producción de semilla de B. alicastrum las técnicas asexuales injerto y acodo pueden ser más eficientes.

\section{Agradecimientos}

Este trabajo forma parte de la Catedra-Conacyt 364 titulada: Reconversión productiva sustentable para el desarrollo de los productores rurales de Campeche. Se agradece a las autoridades del Instituto Tecnológico Superior de Venustiano Carranza por las facilidades brindadas para la defensa de tesis de la Licenciatura en Ingeniería Forestal del segundo autor.

\section{Referencias}

Alba, J. y col. (2017). «Injertos de fenotipos selectos de Pinus greggii engelm. de una prueba genética en Villa Aldama, Veracruz, México». En: Foresta Veracruzana 19.2, 57-61. Online: https: / / bit.ly / 3lokErO.

Bailey, J., K. Bímová y B. Mandák (2009). «Asexual spread versus sexual reproduction and evolution in Japanese Knotweed s.l. sets the stage for the "Battle of the Clones"». En: Biological Inva- 
sions 11.5, 1189-1203. Online: https: / / bit.ly / $33 \mathrm{Cgd} 6 \mathrm{v}$.

Börner, Katy (2011). «Science of Science Studies: Sci2 Tool». En: Communications of the ACM 54.3, 60-69. Online: https://bit.ly/2S2A7ED.

Bouchet, M. y G. Bastin (2013). «RcmdrPlugin.temis, a graphical integrated text mining solution in R». En: The R Journal 5.1, 188-196. Online: https:/ / bit.ly/3iDUUFW.

De Granda, J. y col. (2005). «Las palabras clave como herramientas imprescindibles en las búsquedas bibliográficas. Análisis de las áreas del sistema respiratorio a través de Archivos de Bronconeumología». En: Archivos de Bronconeumología 41.2, 78-83. Online: https://bit.ly/3f399o9.

Del Amo, S. y col. (2002). Germinación y manejo de especies forestales tropicales. CONAFORCONACYT. Online: https://bit.ly/3y2cxbE.

Domínguez, P. y col. (2019). «Textura, color y aceptación sensorial de tortillas y pan producidos con harina de ramón (Brosimum alicastrum) para incrementar la fibra dietética total». En: Ciencia $\mathcal{E}$ Tecnología Agropecuaria 20.3, 699-719. Online: https://bit.ly/3uCgiCc.

García, C., A. Roque y M. Méndez (2012). «Aprovechamiento del ábol nativo Brosimum alicastrum Swartz (Moraceae) en la Selva Zoque Chiapas, México». En: LACANDONIA 6.1, págs. 71-76.

Gillespie, A., D. Bocanegra y J. Jimenez (2004). «The propagation of Ramon (Brosimum alicastrum Sw.; Moraceae) in Mayan homegardens of the Yucatan peninsula of Mexico». En: New Forests 27.1, 25-38. Online: https://bit.ly/3bhlvaW.

Góngora, R. y col. (2016). «Uso tradicional de la flora y fauna en los huertos familiares mayas en el municipio de Campeche, Campeche, México». En: Ecosistemas y recursos agropecuarios 3.9, 379-389. Online: https://bit.ly/3wfYuxf.

González, A. y col. (2019). «Propagation by cuttings and plant quality in Acer negundo L.» En: Revista mexicana de ciencias forestales 10.51, 224-243. Online: https:/ / bit.ly/3y9iSC6.

Gutiérrez, G. y R. Dirzo (2009). «Remoción de semillas, herbivoría y reclutamiento de plántulas de Brosimum alicastrum (Moraceae) en sitios con manejo forestal contrastante de la selva maya, Quintana Roo, México». En: Boletín de la Sociedad Botánica de México 85, 51-58. Online: https: / / bit.ly/3ylbJ1y.

Hernández, O., S. Vergara y A. Larqué (2014). «Studies on the productivity of Brosimum alicastrum a tropical tree used for animal feed in the $\mathrm{Yu}-$ catan Peninsula». En: Bothalia J. Bothanical Life Sci. Res 44.6, 70-81. Online: https: / / bit. ly / 3hewXbm.

- (2015). «Primeras etapas de crecimiento de Brosimum alicastrum Sw. en Yucatán». En: Revista mexicana de ciencias forestales 6.27, 38-49. Online: https://bit.ly/3bBZHXP.

Jacomy, M. y col. (2014). «ForceAtlas2, a continuous graph layout algorithm for handy network visualization designed for the Gephi software». En: PloS one 9.6, e98679. Online: https: / / bit.ly / 36DghVD.

Laborde, J. e I. Corrales (2012). «Direct seeding of Brosimum alicastrum Sw. (Moraceae) and Enterolobium cyclocarpum (Jacq.) Griseb. (Mimosaceae) in different habitats in the dry tropics of central Veracruz». En: Acta botánica mexicana 100, 107-134. Online: https:/ / bit.ly/3tYx0ur.

Loría, L. y A. Larqué (20015). «The effect of salicylic acid on the growth of seedling roots of Brosimum alicastrum, a perennial tree from the Mexican tropics which produces recalcitrant seeds». En: Sylwan 158.6, 338-346. Online: https: / / bit. ly/3hfGPBA.

Luna, T., T. Landis y R. Dumroese (2012). «Producción de plantas en viveros forestales». En: Consejo Federal de Inversiones, Centro de Investigación y Extensión Forestal Andino Patagónico, y Universidad Nacional de la Patagonia San Juan Bosco. Cap. Contenedores: aspectos técnicos, biológicos y económicos, 78-85. Online: https:/ / bit.ly/3uz9SUA.

Magnitskiy, S. y G. Plaza (2007). «Fisiología de semillas recalcitrantes de árboles tropicales». En: Agronomía colombiana 25.1, 96-103. Online: https: //bit.ly/3hdKxf9.

Molina, M. y col. (2015). «Caracterización morfológica in situ de Ojushte (Brosimum alicastrum Swartz) y su incidencia en la selección de germoplasma de alto potencial nutricional en El Salvador». En: Producción Agropecuaria y Desarrollo Sostenible 3, 61-76. Online: https: / / bit.ly / 3f7TpAg.

Orantes, C. y col. (2013). «Viabilidad y germinación de semillas de tres especies arbóreas nativas de la selva tropical, Chiapas, México». En: Polibotánica 36, 117-127. Online: https: / / bit.ly / $3 \mathrm{tSxWkd}$.

Pardo, D., F. Verdugo y J. Hernández (2002). «Reproducción vegetativa de dos especies arbóreas 
en un manglar de la costa norte del Pacífico mexicano». En: Madera y Bosques 8.2, 57-71. Online: https://bit.ly/3bhu0mm.

Peralta, M. y col. (2017). «Reguladores del crecimiento y sustratos en la propagación vegetativa de Nanche (Malpighia mexicana A. Juss. y Byrsonima crassifolia (L) HBK)». En: Revista Brasileira de Fruticultura 39.3, 1-9. Online: https:/ / bit.ly / 33QkJhc.

Pérez de la Cruz, S. y col. (2012). «Diferencias en crecimiento y desarrollo de plántulas de mojú (Brosimum alicastrum Swartz) en condiciones de vivero». En: LACANDONIA 6.2, págs. 51-57.

Peters, C. y E. Pardo (1982). «Brosimum alicastrum (Moraceae): uses and potential in Mexico». En: Economic Botany 36.2, 166-175. Online: https: / / bit.ly/3o1F4cA.

Ramírez, S. y col. (2017). «El ramón (Brosimum alicastrum Swartz) una alternativa para la seguridad alimentaria en México». En: Agroproductividad 10.1, 80-83. Online: https:/ / bit.ly/33v94Ec.

Rojas, J. y col. (2017). «Utilización del follaje de ramón (Brosimum alicastrum Swarth) en la alimen- tación animal». En: Tropical and Subtropical Agroecosystems 20.3, 363-371. Online: https: / / bit.ly / 3vT7nMZ.

Rueda, A. y col. (2014). «Calidad de planta producida en los viveros forestales de Nayarit». En: Revista mexicana de ciencias forestales 5.22, 58-73. Online: https://bit.ly/33SpJSe.

Sampayo, S. y col. (2016). "Enraizado de miniestacas de Cedrela odorata L.» En: Agrociencia 50.7, 919-929. Online: https://bit.ly/3bAIkqo.

Vallejos, J. y col. (2010). «Metodología para la selección e incorporación de árboles plus en programas de mejoramiento genético forestal». En: Agronomía Costarricense 34.1, 105-119. Online: https:/ / bit.ly/3hyGw55.

Vergara, S. y col. (2014). Publicaciones de Brosimum alicastrum. Mérida, Yucatán, México: Centro de Investigaciones Cientificas de Yucatán.

White, D. y C. Hood (2004). «Vegetation patterns and environmental gradients in tropical dry forests of the northern Yucatan Peninsula». En: Journal of Vegetation Science 15.2, 151-160. Online: https:/ / bit.ly/3vTkzBI. 